\title{
Neferine treatment enhances the TRAIL-induced apoptosis of human prostate cancer cells via autophagic flux and the JNK pathway
}

\author{
UDDIN MD NAZIM*, HONGHUA YIN ${ }^{*}$ and SANG-YOUEL PARK \\ Biosafety Research Institute, College of Veterinary Medicine, Jeonbuk National University, \\ Iksan, Jeonbuk 54596, Republic of Korea
}

Received June 30, 2019; Accepted February 18, 2020

DOI: 10.3892/ijo.2020.5012

\begin{abstract}
Prostate cancer (PCa) is a common type of cancer among males, with a relatively high mortality rate. Tumor necrosis factor-related apoptosis-inducing ligand (TRAIL), a member of the tumor necrosis factor (TNF) family, initiates the apoptosis of certain cancer cells. Neferine, a primary ingredient of bisbenzylisoquinoline alkaloids, has various antitumor activities. The present study examined the effects of neferine treatment on human PCa cells. Human prostate cancer (DU145) cells were treated with neferine for $18 \mathrm{~h}$, and subsequently treated with TRAIL for $2 \mathrm{~h}$. Combined treatment with neferine and TRAIL significantly decreased cell viability compared to treatment with TRAIL alone. Furthermore, neferine treatment decreased the expression of p62 and increased LC3B-II expression, as assessed by western blot analysis and immunocytochemistry. It was alsp demonstrated that neferine and TRAIL act synergistically to trigger autophagy in $\mathrm{PCa}$ cells, as revealed by autophagosome formation, LC3B-II accumulation demonstrated by transmission electron microscopy (TEM) analysis and phosphorylated c-Jun N-terminal kinase (p-JNK) upregulation. When the autophagic flux was attenuated by the inhibitor, chloroquine, or by genetically modified ATG5 siRNA, the enhancement of TRAIL-induced autophagy by neferine-induced was also attenuated. Furthermore, treatment with the JNK inhibitor, SP600125, distinctly increased the viability of the cells treated with neferine and TRAIL. On the whole, the findings of the present study demonstrate that neferine treatment effectively
\end{abstract}

Correspondence to: Professor Sang-Youel Park, Biosafety Research Institute, College of Veterinary Medicine, Jeonbuk National University, Gobongro 79, Iksan, Jeonbuk 54596, Republic of Korea

E-mail: sypark@chonbuk.ac.kr

*Contributed equally

Key words: neferine, autophagy, JNK, TRAIL, apoptosis promotes TRAIL-mediated cell death and this effect likely occurs via the autophagic flux and the JNK pathway.

\section{Introduction}

Prostate cancer (PCa) is one of the most frequently occurring malignant tumors among males, and is associated with high mortality and morbidity rates $(1,2)$. To date, no standard regimen for PCa treatment has been established. Chemotherapy, immunotherapy and radiotherapy are the primary cancer treatments used following surgery (3). For patients with $\mathrm{PCa}$, one goal of treatment is to initiate the apoptosis of cancer cells $(4,5)$.

Tumor necrosis factor (TNF)-related apoptosis-inducing ligand (TRAIL), a member of the TNF protein family, is known to trigger the death of various cancer cells, but not normal cells $(6,7)$, by binding the death receptors, DR-4 and DR-5, and by recruiting FADD and caspase- 8 to create a death-inducing complex (8). TRAIL has been used as an apoptosis-inducing factor in various human cancers, and is thus a good candidate for use in novel cancer therapies (9). However, numerous cancer cells acquire resistance to TRAIL-induced apoptosis (5). Thus, for the treatment of $\mathrm{PCa}$, considerable attention has recently been focused on overcoming the resistance of cancer cells to TRAIL.

Neferine is a major bisbenzylisoquinoline alkaloid present in Nelumbo nucifera Gaertn. green seed embryos (10). Recently, neferine has been demonstrated to exhibit efficient antitumor activities in HepG2 cells and human lung cancer cells $(11,12)$, and to suppress the propagation of osteosarcoma cells (13). Further, neferine treatment has been shown to induce the release of reactive oxygen species (ROS) and trigger the mitochondrial apoptosis of liver and lung cancer cells $(11,12)$.

The autophagic flux, which involves the degradation and recycling of damaged and harmful cellular components, is an important process for maintaining metabolism and energy homeostasis (14). Apoptosis leads to programmed cell death, whereas the autophagic flux can lead either to survival or death (15). During the induction of the autophagic flux, beclin-1 triggers the transformation of cytosolic microtubule-associated protein 1A/1B-light chain 3 (LC3-I) into LC3-phosphatidylethanolamine conjugate (LC3-II). The conversion of LC3-I to LC3-II and the recruitment of 
p62/SQTMI to the autophagosomal membrane are considered to be key features of the autophagic flux and are indicators that this process has been induced and activated (16-18), although the specific molecular pathways for this process in cancer cells remain unclear. c-Jun N-terminal kinase (JNK) is a stress-induced member of the mitogen-activated protein kinase (MAPK) family. JNK plays fundamental roles in cell growth, differentiation, attenuation and apoptosis (19). In the present study, the ability of neferine treatment to enhance the TRAIL-initiated apoptosis of PCa cells was assessed. The results indicate that combined treatment of PCa cells with and neferine and TRAIL is more effective than treatment with either substance alone.

\section{Materials and methods}

Cells and cell culture. Human PCa cells (DU145 and LNCaP; Korean Cell Line Bank) were maintained in RPMI-1640 medium containing $10 \%$ fetal bovine serum (FBS). During experimentation, cells were grown in RPMI-1640 (Gibco; Thermo Fisher Scientific, Inc.) containing 1\% FBS. Cells were grown at $37^{\circ} \mathrm{C}$ and $5 \% \mathrm{CO}_{2}$ in a humidified incubator.

Reagents. Neferine was acquired from Sigma-Aldrich; Merck $\mathrm{KGaA}$, and TRAIL was acquired from Abfrontier.

Determination of cell cytotoxicity. Cytotoxicity is the amount of toxiciticy affecting cells. A cytotoxic agent can lead to a decrease in cell viability, the activation of apoptosis and the alteration of autophagy (20-22). Several methods for cytotoxicity assay, such as trypan blue stain, lactate dehydrogenase (LDH) assay, 3-(4,5-dimethyl-2-thiazoly)-2,5-dephenyl-2H-tetrazolium bromide (MTT) assay. In the present study, cell viability was assessed, and crystal violet and trypan blue staining was used to examine cells treated with a combination of neferine and TRAIL.

Cell viability assay. The DU145 and LNCaP cells seeded in 12 -well plates were treated with $0,5,10$, or $20 \mu \mathrm{M}$ neferine for $18 \mathrm{~h}$, cells were subseqently treated with $200 \mathrm{ng} / \mathrm{ml} \mathrm{TRAIL}$ for $2 \mathrm{~h}$ and chloroquine (CQ; cat. no. c6628; Sigma-Aldrich; Merck KGaA) was treated $10 \mu \mathrm{M} 1 \mathrm{~h}$ prior neferine or TRAIL treatment. In addition, the JNK inhibitor, SP600125 (cat. no. s5567; Sigma-Aldrich; Merck KGaA), was used to treat the cells at $1 \mu \mathrm{M}$ for $1 \mathrm{~h}$ prior to neferine or TRAIL treatment. Cell morphology was evaluated using a light microscope (Nikon Corp.), and cell viability was evaluated using a crystal violet assay as previously described (23).

Trypan blue exclusion assay. Cell viability was evaluated using a trypan blue exclusion assay. The DU145 and LNCap cells were seeded in a 24 -well plate and following treatment, the cells were dissociated from plate with trypsin-EDTA and were then suspensed in $1 \mathrm{ml}$ PBS per well followed by the addition of $1 \mathrm{ml}$ trypan blue solution (Sigma-Aldrich; Merck $\mathrm{KGaA}$ ). Follwing incubation for $5 \mathrm{~min}$ at $20^{\circ} \mathrm{C}$, the cells were counted using a hemocytometer (Marienfeld Corp.) and examined using a light microscope (Nikon Corp.). Each treatment was performed in triplicate, and the results are expressed as a percentage relative to the untreated controls.
Immunofluorescence staining. To determine the effects of neferine on TRAIL-induced apoptosis, the levels of cleaved casapase-8 (Ac-cas8) and caspase-3 (Ac-cas3) were detected. In addition, Also, changes in the levels of p62 and LC-3 II proteins were detected to monitor the autophagic flux. DU145 cells were cultured on poly-L-lysine-coated coverslips. Differentiated cells that had been treated were fixed with $4 \%$ paraformaldehyde and permeabilized with $0.1 \%$ Triton X-100. The cells were then incubated in blocking solution overnight at $4^{\circ} \mathrm{C}$ and bathed in a solution containing anti-p62 (1:250; cat. no. PAS-20839; Invitrogen; Thermo Fisher Scientific, Inc.) and anti-p-JNK (1:500; cat. no. c.s 9255s; Cell Signaling Technology; Inc.) antibodies for 2-3 h at room temperature. After washing with PBS, the cells were incubated in the dark at $20^{\circ} \mathrm{C}$ with secondary antibodies (Alexa Fluor ${ }^{\circledR} 488$-conjugated donkey polyclonal anti-rabbit; 1:500; cat. no. A-21206; Thermo Fisher Scientific, Inc. and Alexa FluorTM 546 goat anti-mouse IgG (1:500; cat. no. A-21206; Thermo Fisher Scientific, Inc.) for $2 \mathrm{~h}$. A fluorescence microscope (Nikon Corp.) was used to visualize immunostaining.

Transmission electron microscopy (TEM). Cells were bathed in a solution containing $2 \%$ glutaraldehyde, $2 \%$ paraformaldehyde and $0.05 \mathrm{M}$ sodium cacodylate $(\mathrm{pH}$ 7.2) (all from Electron Microscopy Sciences) for $2 \mathrm{~h}$ at $4^{\circ} \mathrm{C}$. The cells were then fixed by incubation with $1 \%$ osmium tetroxide (Electron Microscopy Sciences) for $1 \mathrm{~h}$ at $4^{\circ} \mathrm{C}$, dehydrated with increasing concentrations of ethanol $(25,50,70,90$ and $100 \%$ ) for $5 \mathrm{~min}$ at each concentration, and embedded in epoxy resin (Embed 812; Electron Microscopy Sciences) for $48 \mathrm{~h}$ at $60^{\circ} \mathrm{C}$ following the manufacturers' instructions. Ultrathin sections (60-nm-thick) were sliced using an LKB-III ultratome (Leica Microsystems $\mathrm{GmbH}$ ). The slices were stained with $0.5 \%$ uranyl acetate (Electron Microscopy Sciences) for $20 \mathrm{~min}$ and with $0.1 \%$ lead citrate (Electron Microscopy Sciences) for $7 \mathrm{~min}$ at room temperature. Fluorescent images were recorded using the Hitachi H7650 electron microscope (Hitachi, Ltd.; magnification, x10,000) located at the Center for University-Wide Research Facilities (CURF) at Jeonbuk National University.

Western blot analysis. Western blot analysis was performed as previously described (3). Total protein extraction was using immunoprecipitation assay buffer (Qiagen, Inc.). The supernatant was collected by centrifugation $(11,200 \mathrm{x} \mathrm{g})$; $4^{\circ} \mathrm{C} ; 10 \mathrm{~min}$; the protein concentration was determined using the BCA protein assay kit (Thermo Fisher Scientific; Inc.). Proteins $(30 \mu \mathrm{g})$ were separated on $10 \%$ SDS-PAGE gels and blotted onto polyvinylidene fluoride membranes. The membranes were blocked with $5 \%$ non-fat dried milk at $25^{\circ} \mathrm{C}$ for $1 \mathrm{~h}$ antibodies against the flowing proteins were used; $\beta$-actin (1:10,000; cat. no. A5441; Sigma-Aldrich; Merck KGaA), LC3A/B (1:1,000; cat. no. c.s 4108s; Cell Signaling Technology; Inc.), p62 (1:250; cat. no. PAS-20839; Invitrogen; Thermo Fisher Scientific, Inc.), ATG5 (1:1,000; cat. no. c.s 12994s; Cell Signaling Technology; Inc.), cleaved caspase-3 (1:1,000; cat. no. c.s 9661; Cell Signaling Technology; Inc.) (activation of caspase-3 requires proteolytic processing of its inactive zymogen into activated p17 and p12 fragments, cleavage of caspase-3 requires aspartic acid at 
A

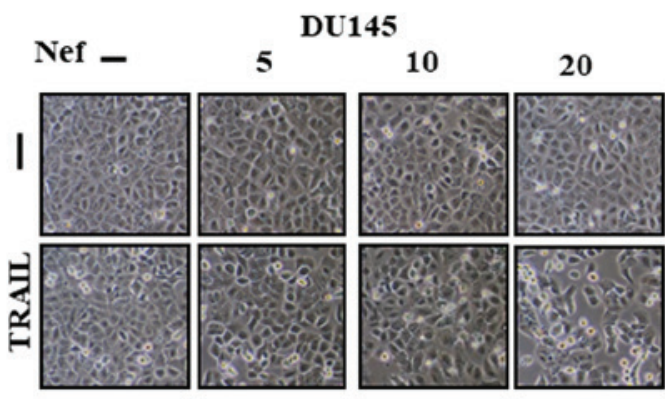

B

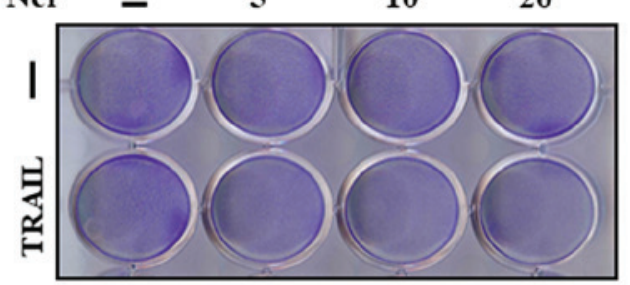

C
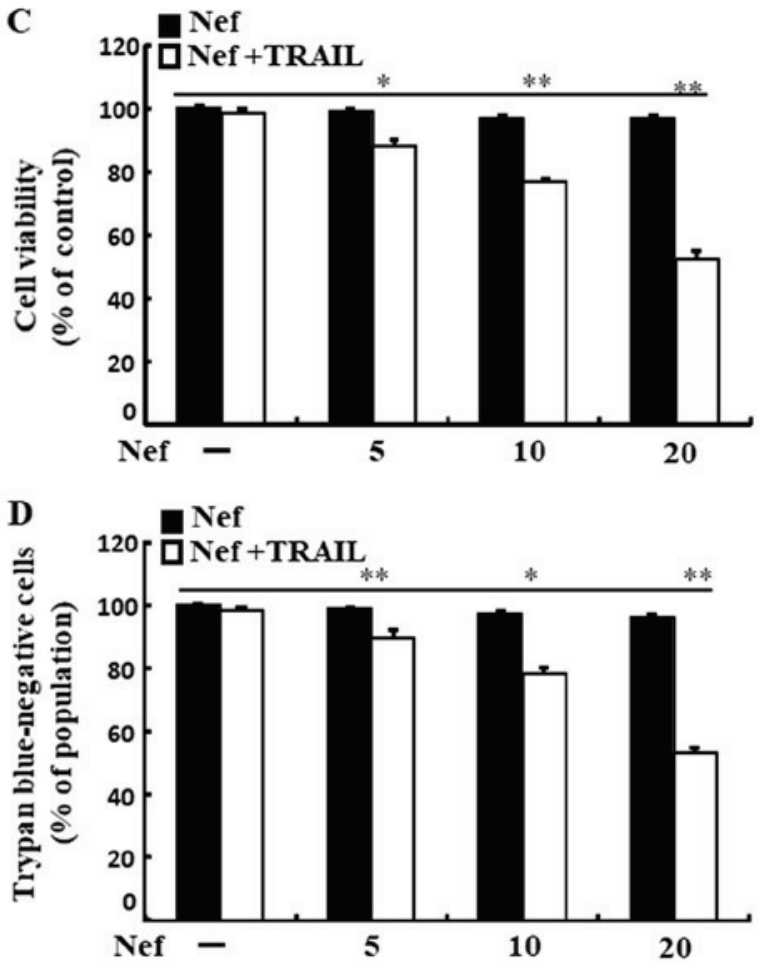

$\mathbf{E}$

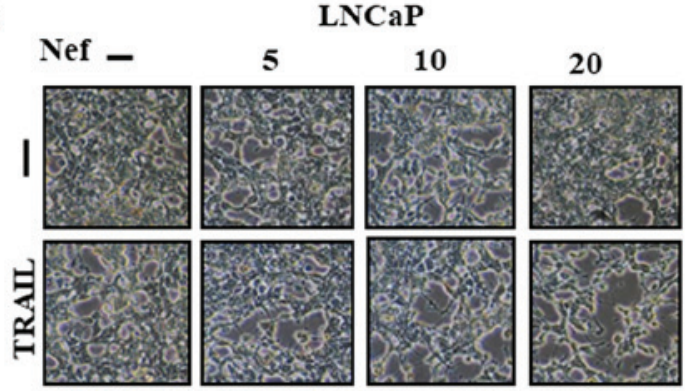

F Nef $-5 \quad 5 \quad 10 \quad 20$

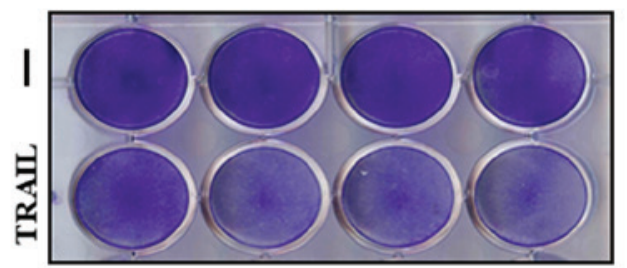

G

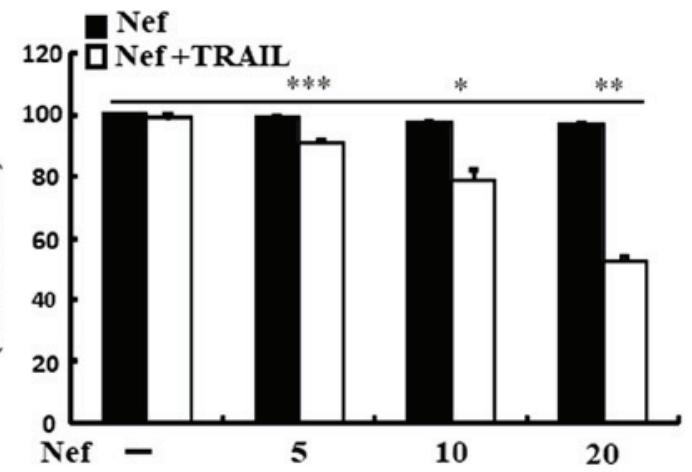

H

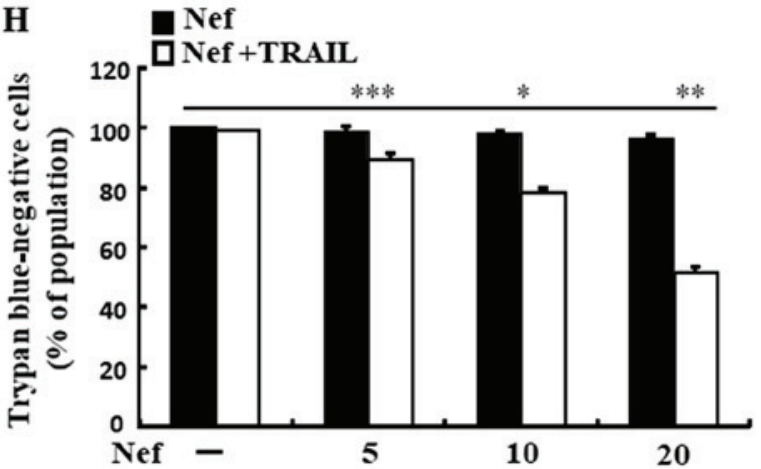

Figure 1. Effect of neferine on the TRAIL-triggered apoptosis of prostate cancer cells. (A and E) DU145 and LNCaP cells were incubated with neferine at concentrations of $0,5,10$ or $20 \mu \mathrm{M}$ for $18 \mathrm{~h}$, and were then exposed to TRAIL at a concentration of $200 \mathrm{ng} / \mathrm{ml}$ for $2 \mathrm{~h}$. The morphology of the cells was evaluated by light microscopy. (B and F) Cell viability was determined by crystal violet staining. (C and G) Bar graphs illustrate the dyed crystal violet average densities. ( $\mathrm{D}$ and $\mathrm{H}$ ) Cell viability was also assessed by trypan blue exclusion assays. ${ }^{*} \mathrm{P}<0.05,{ }^{* *} \mathrm{P}<0.01,{ }^{*}{ }^{* * *} \mathrm{P}<0.001$ indicate significance for comparisons between the treatment and untreated control groups. Nef, neferine; TRAIL, tumor necrosis factor (TNF)-related apoptosis-inducing ligand.

thep1 position), cleaved caspase-8 (1:1,000; cat. no. 551242; BD Pharmingen) (caspase- 8 is produced as a proenzyme $(55 / 50$ $\mathrm{kDa}$, doublet) it is cleaved into smaller subunits $(40 / 36 \mathrm{kDa}$, doublet). and p-JNK (1:500; cat. no. c.s 9255s; Cell Signaling Technology; Inc.). The membranes were then incubated with horseradish peroxidase-conjugated secondary antibodies (cat. nos. ADI-SAB-100 and ADI-SAB-300; 1:1,000; Enzo Life Sciences, Inc.) at $25^{\circ} \mathrm{C}$ for $1 \mathrm{~h}$. The immune reactive protein bands were visualized using enhanced chemiluminescence detection system (GE Healthcare Life Sciences) and detected with chemiluminescence imaging system (Fusion FX7; Viber
Lourmat). The intensities of the protein bands were determined using Image J Java 1.8.0 software.xs.

Small interfering RNA transfection. Media RPMI-1640 (Gibco; Thermo Fisher Scientific, Inc.) containing 10\% FBS (Atlas Biologicals) was seeded with DU145 cells, and $24 \mathrm{~h}$ later, the cells were transfected with silencer-select small interfering RNA (ATG5 siRNA; oligo ID HSS114103; Sequence GGU UUGGACGAAUUCCAACUUGUUU; Invitrogen; Thermo Fisher Scientific, Inc.) using Lipofectamine 2000 as per the manufacturer's recommendations. Simultaneously, a negative 

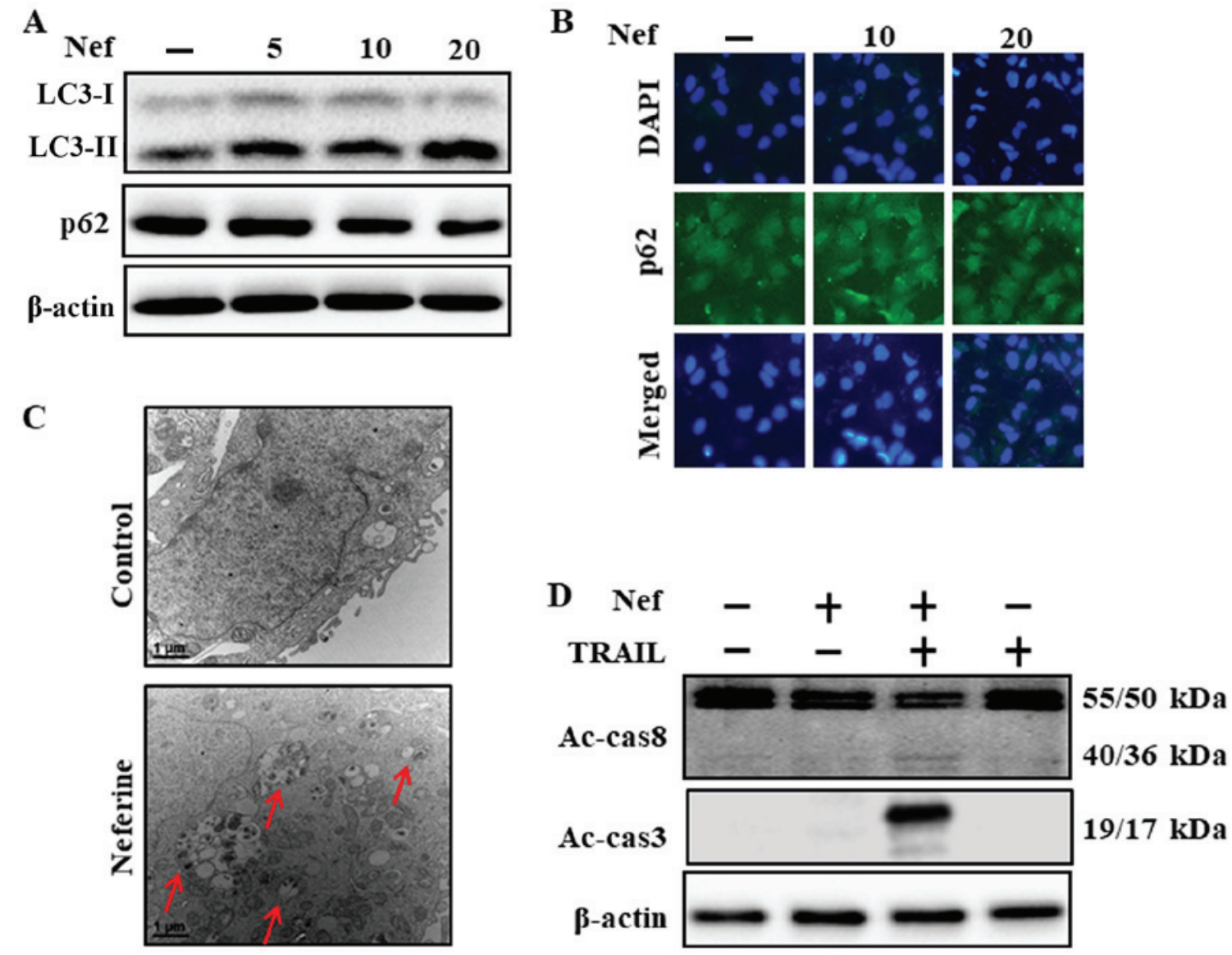

Figure 2. Neferine stimulates the autophagic flux and triggers TRAIL-induced apoptosis. (A and D) DU145 cells were incubated with $20 \mu \mathrm{M}$ neferine for $18 \mathrm{~h}$ and were then incubated with TRAIL for $1 \mathrm{~h}$. p62, LC3-II, Ac-cas3 and Ac-cas8 levels were evaluated by western blot analysis and normalized to $\beta$-actin levels. (B) Cells were immunostained with p62 (green) and counterstained with DAPI (blue). (C) The construction of autophagosomes in treated cells was evaluated by transmission electron microscopy. Nef, neferine; TRAIL, tumor necrosis factor (TNF)-related apoptosis-inducing ligand.

control (Invitrogen; Thermo Fisher Scientific, Inc.) was transfected with a non-targeting siRNA. The cells were incubated with ATG5 siRNA or negative control siRNA for $6 \mathrm{~h}$ and the medium was then changed to RPMI-1640 with $10 \%$ FBS for $24 \mathrm{~h}$. The cells were then treated with neferine or neferine in combination with TRAIL.

Statistical analysis. All experiments were performed in triplicate, and the data are reported as the means \pm standard error. One-way factorial analysis of variance (ANOVA), followed by Duncan's post-hoc test, was performed to evaluate the statistical significance of the differences between the treatment and control groups.

\section{Results}

Effects of neferine treatment on the TRAIL-induced apoptosis of PCa cells. In the present study, in order to investigate the synergistic effects of combination treatment with neferine and TRAIL on PCa cells, the viability of neferine only-treated cells, TRAIL only-treated cells, and neferine and TRAIL combine-treated cells was compared. No significants changes were observed in the viability of the cells treated with neferine or TRAIL only; however, a significant decrease was detected in the viability of the cells treated with the combination of neferine and TRAIL (Fig. 1). To evaluate the effects of neferine treatment on PCa cell apoptosis, the DU145 and LNCaP cells were treated with various concentrations of neferine for $18 \mathrm{~h}$ and then exposed to TRAIL for a further $2 \mathrm{~h}$. As shown in Fig. 1, treatment with TRAIL or neferine alone resulted in only marginal cell death and did not cause any novel morphological changes. By contrast, when cells were treated with both neferine and TRAIL, cell viability decreased significantly (Fig. 1). These data indicate that neferine treatment sensitizes human PCa cells to TRAIL-induced apoptosis.

Neferine treatment induces an autophagic flux and promotes TRAIL-mediated apoptosis. In response to neferine treatment, the expression of LC3-II increased markedly in the DU145 cells, whereas p62 expression decreased significantly (Fig. 2A). The results obtained for $\mathrm{p} 62$ protein levels measured by immunofluorescence staining were consistent with those obtained by western blot analysis (Fig. 2B). TEM indicated that autophagic vacuoles were secreted by the neferine-treated cells (Fig. 2C). In addition, the cells treated with a combination of neferine and TRAIL exhibited higher expression levels of Ac-cas3 and Ac-cas8 (Fig. 2D). Caspase-8 activation also was detected in the cells treated with a combination of neferine and TRAIL, but not in the cells treated with neferine or TRAIL alone (Fig. 2D). Certain studies have compared the combination of two agents $\S$ to either agent alone to investigate the synergistic effects of the two agents (24-26). These 

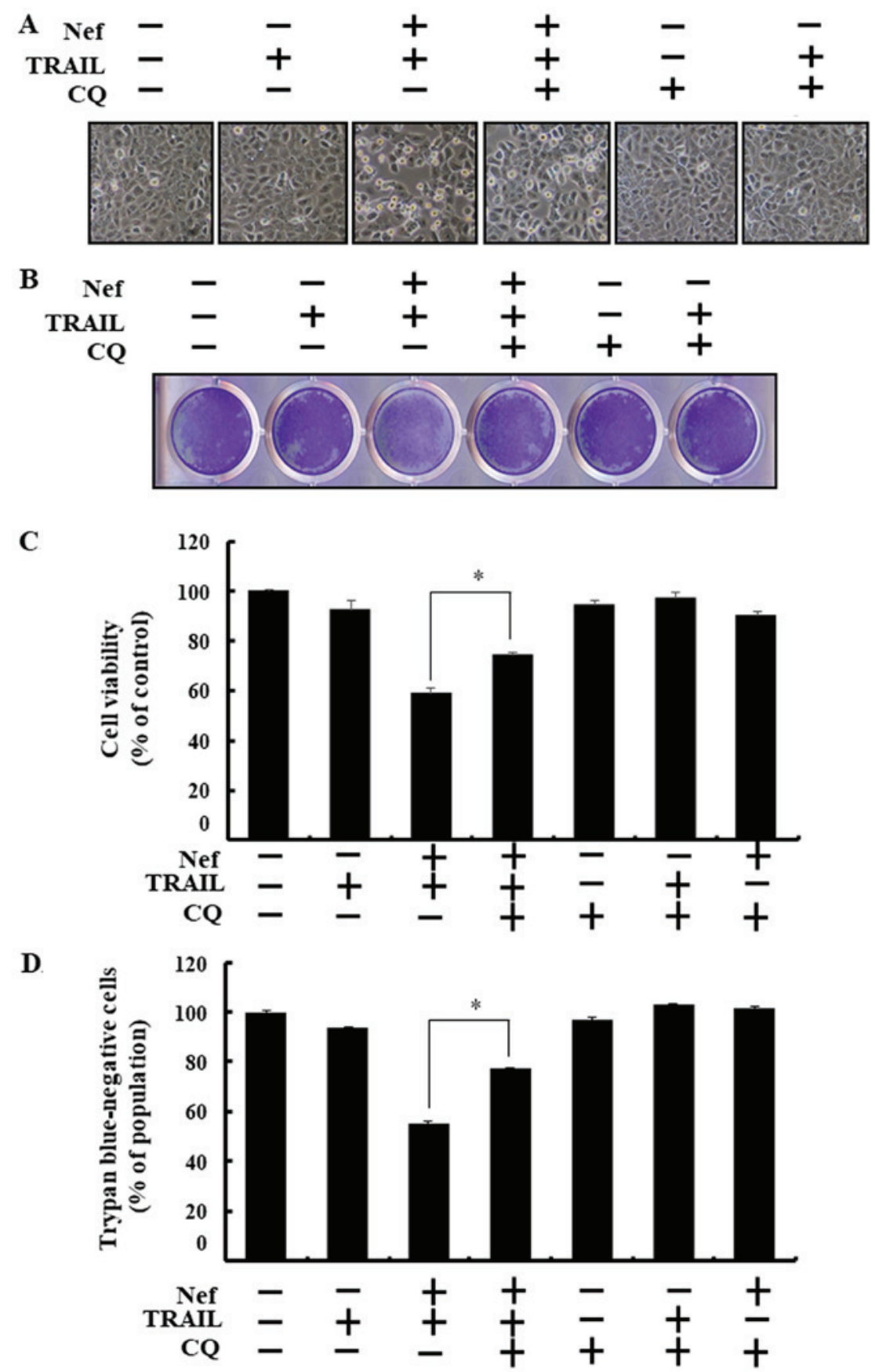

Figure 3. Neferine-mediated, TRAIL-triggered apoptosis is suppressed when autophagy is inhibited. DU145 cells were treated with CQ prior to exposure to $20 \mu \mathrm{M}$ neferine for $18 \mathrm{~h}$ and to TRAIL for $2 \mathrm{~h}$. (A) The morphology of the cells was assessed by light microscopy. (B) Cell viability was determined by crystal violet staining. (C) Bar graph presents the average dyed crystal violet densities. (D) Cell viability was also determined by trypan blue exclusion assays. "P<0.05, indicates significance for comparisons between the untreated control and treatment groups. Nef, neferine; TRAIL, tumor necrosis factor (TNF)-related apoptosis-inducing ligand; $\mathrm{CQ}$, chloroquine.

results suggest that neferine treatment can initiate autophagy in DU145 cells.

Inhibition of autophagy attenuates the neferine-mediated sensitization of TRAIL-induced apoptosis. Cell morphological analysis revealed that treatment with chloroquine (CQ), an autophagy inhibitor, attenuated cellular apoptosis mediated by combined treatment with neferine and TRAIL (Fig. 3A). Co-treatment with neferine, TRAIL and CQ resulted in a distinct improvement in the viability of the DU145 cells (Fig. 3B-D). The autophagic flux activation by neferine was confirmed by the inspection of the autophagic flux following treatment with CQ as an autophagy inhibitor. CQ treatment led to the accumulation of membrane-bound LC3-II and an increase in p62 levels, these results indicated that CQ blocked neferine-induced autophagy (Fig. 4A). Immunofluorescence staining indicated that $\mathrm{CQ}$ treatment resulted in an increase in p62 protein levels (Fig.4B). CQ also attenuated the upregulation of Ac-cas8 and Ac-cas3 that was observed following treatment with neferine and TRAIL (Fig. 4C). These results indicate that CQ modulates neferine-mediated, TRAIL-triggered apoptosis by inhibiting the autophagic flux. 

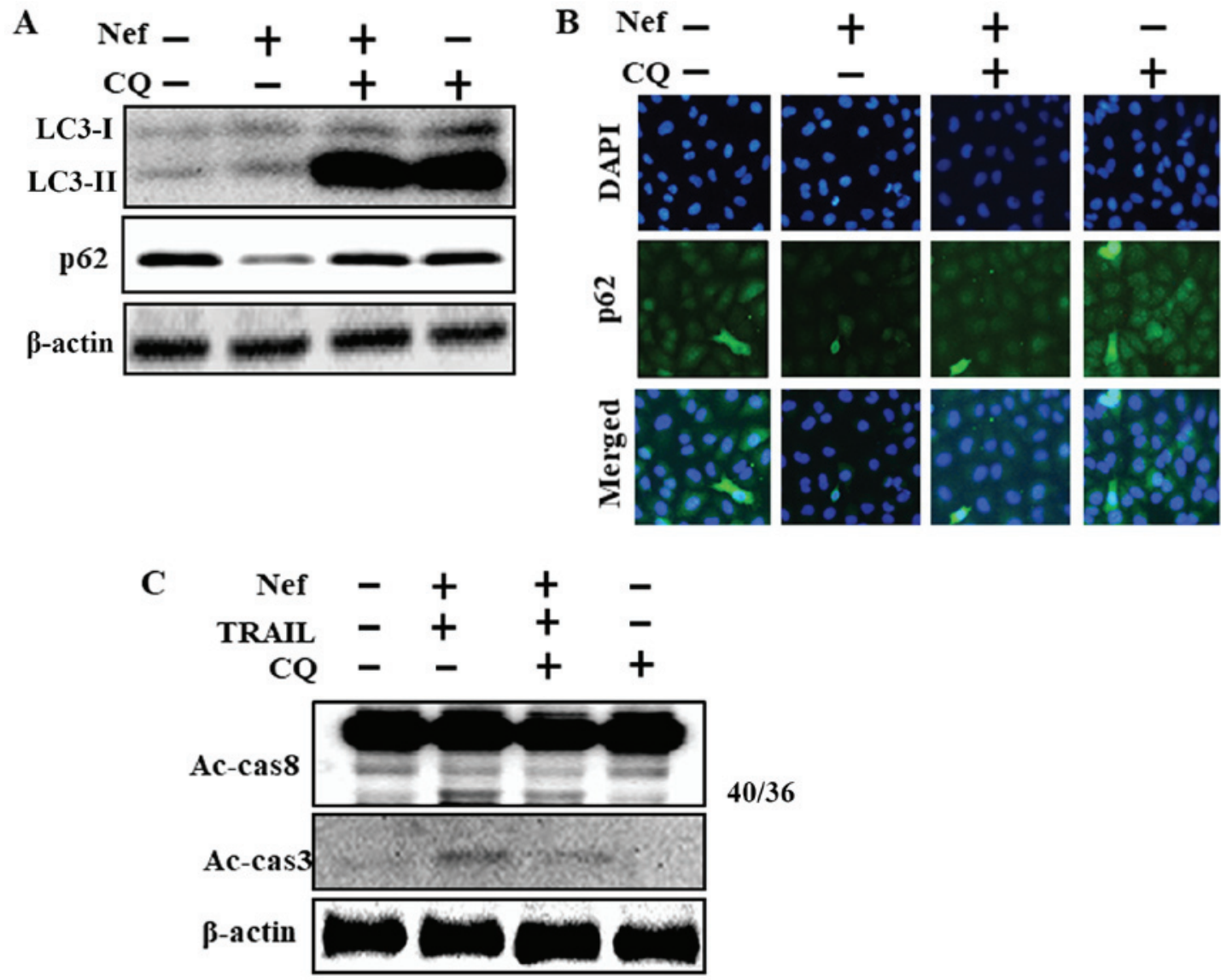

$40 / 36$

Figure 4. Neferine mediates sensitization to TRAIL-triggered apoptosis by promoting the autophagic flux. DU145 cells were treated with CQ prior to exposure to $20 \mu \mathrm{M}$ neferine for $18 \mathrm{~h}$, followed by exposure to TRAIL for $1 \mathrm{~h}$. (A and C) p62, LC3-II, Ac-cas3 and Ac-cas 8 expression levels were evaluated by western blot analysis. (B) Cells were immunostained with p62 (green) and counterstained with DAPI (blue). Nef, neferine; TRAIL, tumor necrosis factor (TNF)-related apoptosis-inducing ligand; $\mathrm{CQ}$, chloroquine.

Synergistic apoptosis mediated by neferine and TRAIL is blocked by the genetic inhibition of the autophagic flux. Cell morphological analysis indicated that transfection with ATG5 siRNA (Fig. 5A). Co-treatment with neferine, TRAIL and ATG5 siRNA significantly attenuated cell death and markedly increased the viability of the DU145 cells (Fig. 5B-D). In the cells in which ATG5 was knocked down, the LC3-II protein levels were markedly decreased and p62 protein levels were markedly increased (Fig. 6A). The protein levels of p62 measured by immunofluorescence staining were similar to the levels measured by western blot analysis (Fig. 6B). Co-treatment of the cells with neferine, ATG5 siRNA and TRAIL resulted in a decrease in Ac-cas3 and Ac-cas8 expression (Fig. 6C). These results suggested that ATG5 siRNA blocked the synergistic cell death induced by neferine and TRAIL treatment.

Neferine treatment induces JNK activation. As revealed by western blot analysis and immunofluorescence staining, neferine treatment of the DU145 cells resulted in a dose-dependent increase in the $\mathrm{p}-\mathrm{JNK}$ protein expression levels (Fig. 7A and B). In the cells that were treated with $1 \mu \mathrm{M}$ SP600125 for $1 \mathrm{~h}$ prior to neferine treatment, a decrease in neferine-induced p-JNK expression was observed. SP600125 treatment also increased the viability of the cells that had been treated with neferine and TRAIL (Fig. 7D and E). These results indicate that neferine treatment causes an increase in JNK expression that triggers TRAIL-mediated death of DU145 cells.

\section{Discussion}

TRAIL has been demonstrated to stimulate the apoptosis of cancer cells without harming normal cells; therefore, TRAIL administration is regarded as a prospective treatment strategy against cancer (27-29). However, TRAIL resistance has been observed in a number of different types of cancer (30). Neferine is a bisbenzyl isoquinoline alkaloid that has been shown to exert a number of biological effects, such as the inhibition of cancer cell proliferation (31). The autophagic flux, a process of lysosomal degradation of misfolded and unneeded proteins, plays a crucial role in maintaining homeostasis in healthy cells, and can also lead to the destruction of damaged or cancerous cells (32-34). JNK plays a critical role in inducing autophagy and triggering cellular apoptosis (35). Cell viability is the ratio of the initial cell number minus the dead cell number to the initial cell number. In the present study, cell viability assay was used to assess apoptotic cell death. Cleaved caspase- 3 and caspase- 8 were also detected for the assessment of apoptosis (Figs. 2D, 4C and 6C).

TRAIL has received considerable attention as a novel anticancer agent. Although various types of tumor cells 

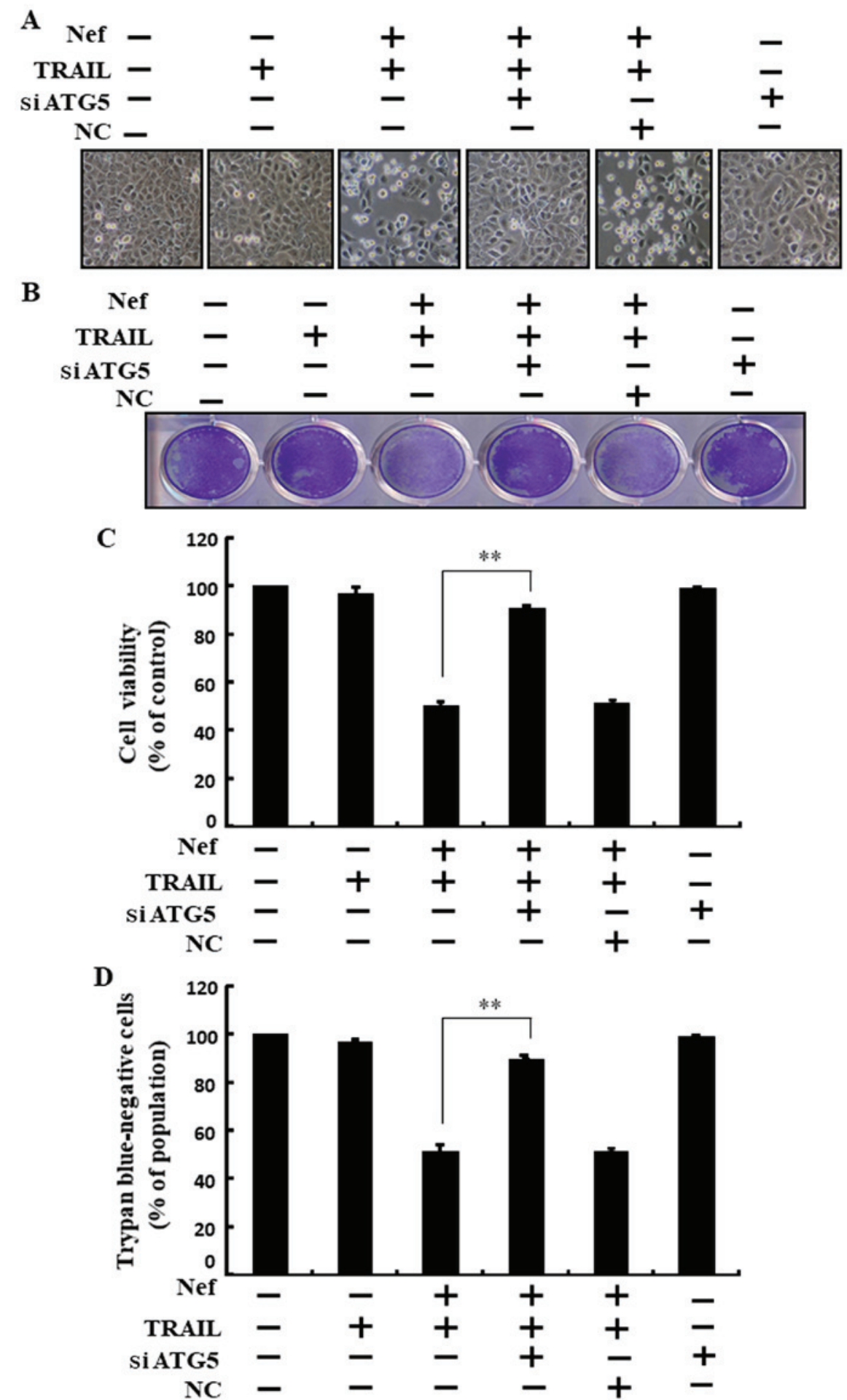

Figure 5. Neferine mediates TRAIL-triggered apoptosis by the genetic attenuation of autophagy. DU145 cells were transfected with ATG5 siRNA or negative control siRNA for $24 \mathrm{~h}$ prior to exposure to $20 \mu \mathrm{M}$ neferine for $18 \mathrm{~h}$ and to TRAIL for $2 \mathrm{~h}$. (A) The morphology of the cells was assessed by light microscopy. (B) Cell viability was determined by crystal violet assay. (C) Bar graph presents the average densities of dyed crystal violet. (D) Cell viability was also determined using a trypan blue exclusion assay. ${ }^{* *} \mathrm{P}<0.01$, indicates significance for comparisons between the untreated control and treatment groups. Nef, neferine; TRAIL, tumor necrosis factor (TNF)-related apoptosis-inducing ligand.

are sensitive to TRAIL-initiated apoptosis, other cells, including PCa cells, are TRAIL-resistant (36). The data of the present study demonstrated that while TRAIL treatment alone did not trigger the apoptosis of DU145 cells, treatment of the cells with neferine prior to TRAIL treatment resulted in increased cell death (Fig. 1). Recent research has revealed that neferine attenuates cancer cell prolifera- tion and induces autophagy $(12,31,37)$. The present study demonstrated that neferine initiated autophagy in PCa cells through the formation of autophagosomes and the conversion of LC3-I to LC3-II (Fig. 2), and that pharmacological (Figs. 3 and 4) and genetic (Figs. 5 and 6) autophagy inhibitors attenuated neferine-mediated, TRAIL-triggered apoptosis. 

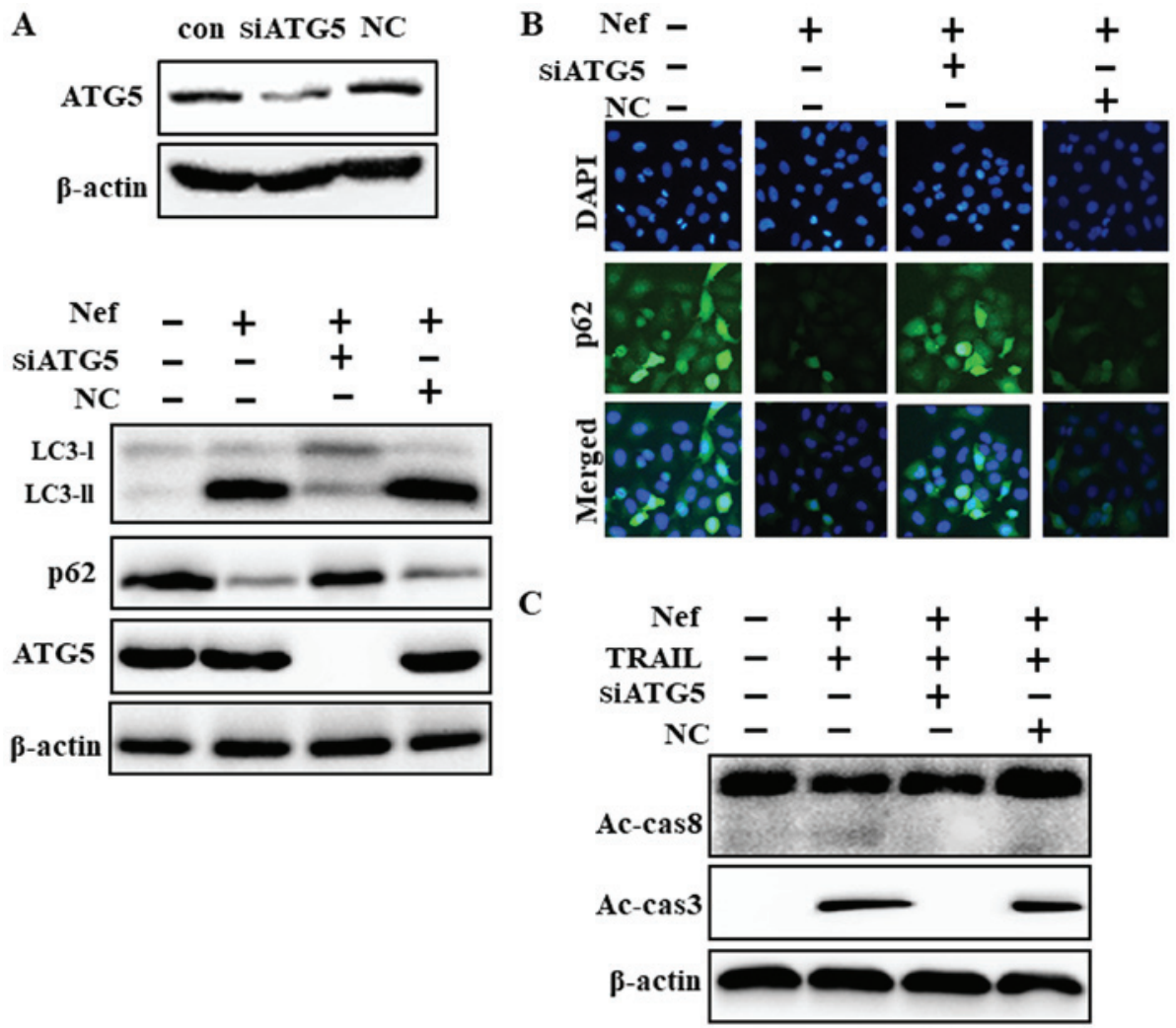

Figure 6. Neferin mediates TRAIL-triggered apoptosis by activating the autophagic flux. DU145 cells were transfected with ATG5 siRNA (siATG5) or negative control siRNA (siNC) for $24 \mathrm{~h}$ prior to exposure to $20 \mu \mathrm{M}$ neferine for $18 \mathrm{~h}$, and to TRAIL for $1 \mathrm{~h}$. (A and C) p62, LC3-II, ATG5, Ac-cas3 and Ac-cas8 levels were evaluated by western blot analysis and (B) cells were immunostained with p62 antibody (green) and counterstained with DAPI (blue). Nef, neferine; TRAIL, tumor necrosis factor (TNF)-related apoptosis-inducing ligand.
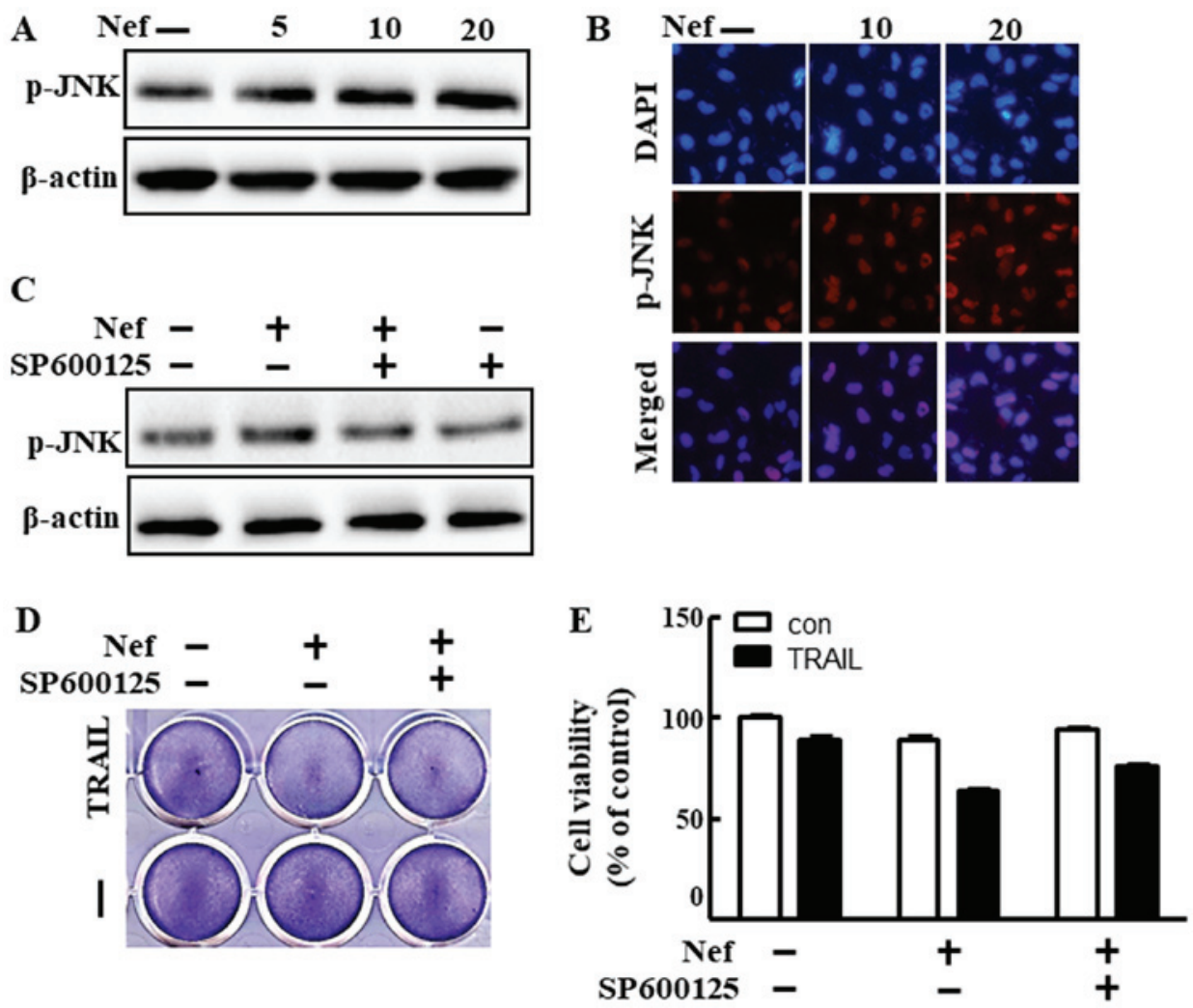

Figure 7. Neferine activates the JNK pathway. DU145 cells were treated with neferine $(0,5,10$ and $20 \mu \mathrm{M})$ for $18 \mathrm{~h}$. (A and B) p-JNK protein levels were evaluated by western blot analysis and immunofluorescence assay. (C) DU145 cells were treated with SP600125 for 1 h prior to exposure to $20 \mu \mathrm{M}$ neferine for $18 \mathrm{~h}$. (D and E) and to $200 \mathrm{ng} / \mathrm{ml}$ TRAIL for $1 \mathrm{~h}$, after which cell viability was assessed using a crystal violet assay. Nef, neferine; TRAIL, tumor necrosis factor (TNF)-related apoptosis-inducing ligand. 
Autophagy and apoptosis are self-destructive processes in response to cell stress. These two processes are activated by different signaling pathway, but also interact to each other. They finally lead to cell death and decrease cell viability, particularly that of cancer cells; however, these two processes have different biomarkers for identification. Apoptosis is programmed cell death. There are certain biomarkers for monitoring apoptosis, such as caspase, mitochondrial potential, the sub G1 population, DNA fragmentation and nuclear condensation (38). Western blot analysis is a powerful method for the detection of apoptosis. During apoptosis the levels of a number of proteins are altered. The levels of caspases, such as caspase- 3 , caspase- 8 and caspase- 9 are significantly increased during apoptosis (39). In addition, the levels of $\mathrm{Bcl}$ family proteins are altered during apoptosis (40). The depolarization of mitochondrial potential is the central mechanism of apoptosis (41). The induction of apoptosis induces DNA fragmentation and nuclear condensation, leading to nuclear morphological changes, and this increases the Sub G1 cell population (42). These methods can identify apoptotic cell death. Autophagy selects and tags cytoplasmic components and organelles into the autophagosome and which are then degraded by the lysosome. There are some available methods with which to detect autophagy structures and monitor the autophagic flux (43). In the present study, LC-3 and p62 protein expression was examined by western blot analysis and immunocytochemistry. LC-3 and p62 are typical protein makers of the autophagic flux (43). LC-3 as an autophagosome marker, is presented in Figs. 2A, 4A and 6A. LC-3 is a microtubule-associated protein $1 \mathrm{~A} / 1 \mathrm{~B}$-light chain, and during autophagy the cytosolic form of LC-3 (LC-3I) is conjugated to phosphatidylethanolamine (PE) to form LC-3-PE (LC-3II). Subsequently, LC-3II contributes to autophagosome formation. LC-3 has been used as a maker of autophagosome formation $(44,45)$. The present study detected p62 as a maker of the autophagic flux (Figs. 2A and B, 4A and B, and 6A and B). p62 is sequestosome-1, and is an ubiquitin-binding protein. It delivers cytosolic protein to the autophagosome and directly binds to LC-3II. p62 allows the cytosolic protein to locate into the autophagosome and be degraded. A decrease in p62 expression can represent an increase in the autophagic flux. In several studies, the expression of p62 has been monitored for the investigation of the autophagic flux $(46,47)$. The present study also detected autophagosome structure using TEM (Fig. 2C). The autophagosome is a double membrane vesicle, substrates some cellular organelles and aggregated proteins. TEM is a conventional method used to identify the autophagosome and monitor the morphology of the autophagosome (48).

The present study also demonstrated that neferine treatment increased p-JNK protein expression in a dose-dependent manner (Fig. 7A and B). The effects of neferine treatment were suppressed in the presence of SP600125, which inhibits p-JNK activity (Fig. 7C and D). In conclusion, the findings of the present study demonstrate that neferine treatment mediates TRAIL-the induced death of DU145 tumor cells via the autophagic flux and JNK pathway. These results indicate that combined treatment with neferine and TRAIL may be effective against TRAIL-resistant cancers.

\section{Acknowledgements}

Not applicable.

\section{Funding}

The present study was supported by the National Research Foundation of the Korea Grant (NRF) funded by the Ministry of Education (2019R1A6A1A03033084).

\section{Availability of data and materials}

All data generated or analyzed during this study are included in this published article or are available from the corresponding author on reasonable request.

\section{Authors' contributions}

UMDN, HY and SYP designed, executed the study and analyzed data. UMDN and HY wrote the manuscript. All authors have read and approved the final manuscript.

\section{Ethics approval and consent to participate}

Not applicable.

\section{Patient consent for publication}

Not applicable.

\section{Competing interests}

The authors declare that they have no competing interests.

\section{References}

1. Siegel R, Naishadham D and Jemal A: Cancer statistics, 2012. CA Cancer J Clin 62: 10-29, 2012.

2. Hao L, Zhao Y, Li ZG, He HG, Liang Q, Zhang ZG, Shi ZD, Zhang PY and Han CH: Tumor necrosis factor-related apoptosis-inducing ligand inhibits proliferation and induces apoptosis of prostate and bladder cancer cells. Oncol Lett 13: 3638-3640, 2017.

3. Nazim UM, Jeong JK and Park SY: Ophiopogonin B sensitizes TRAIL-induced apoptosis through activation of autophagy flux and downregulates cellular FLICE-like inhibitory protein. Oncotarget 9: 4161-4172, 2018.

4. Zhang S, Wang Y, Chen Z, Kim S, Iqbal S, Chi A, Ritenour C, Wang YA, Kucuk O and Wu D: Genistein enhances the efficacy of cabazitaxel chemotherapy in metastatic castration-resistant prostate cancer cells. Prostate 73: 1681-1689, 2013.

5. Klosek M, Mertas A, Krol W, Jaworska D, Szymszal J and Szliszka E: Tumor necrosis factor-related apoptosis-inducing ligand-induced apoptosis in prostate cancer cells after treatment with xanthohumol-a natural compound present in humulus lupulus L. Int J Mol Sci 17: pii: E837, 2016.

6. Wiley SR, Schooley K, Smolak PJ, Din WS, Huang CP, Nicholl JK, Sutherland GR, Smith TD, Rauch C, Smith CA, et al: Identification and characterization of a new member of the TNF family that induces apoptosis. Immunity 3: 673-682, 1995.

7. Wang S and El-Deiry WS: TRAIL and apoptosis induction by TNF-family death receptors. Oncogene 22: 8628-8633, 2003.

8. Kischkel FC, Lawrence DA, Chuntharapai A, Schow P, Kim KJ and Ashkenazi A: Apo2L/TRAIL-dependent recruitment of endogenous FADD and caspase- 8 to death receptors 4 and 5 . Immunity 12: 611-620, 2000.

9. Wei RJ, Zhang XS and He DL: Andrographolide sensitizes prostate cancer cells to TRAIL-induced apoptosis. Asian J Androl 20: 200-204, 2018. 
10. Sivalingam KS, Paramasivan P, Weng CF and Viswanadha VP: Neferine potentiates the antitumor effect of cisplatin in human lung adenocarcinoma cells via a mitochondria-mediated apoptosis pathway. J Cell Biochem 118: 2865-2876, 2017.

11. Poornima P, Quency RS and Padma VV: Neferine induces reactive oxygen species mediated intrinsic pathway of apoptosis in HepG2 cells. Food Chem 136: 659-667, 2013.

12. Poornima P, Weng CF and Padma VV: Neferine, an alkaloid from lotus seed embryo, inhibits human lung cancer cell growth by MAPK activation and cell cycle arrest. Biofactors 40: 121-131, 2014

13. Zhang X, Liu Z, Xu B, Sun Z, Gong Y and Shao C: Neferine, an alkaloid ingredient in lotus seed embryo, inhibits proliferation of human osteosarcoma cells by promoting p38 MAPK-mediated p21 stabilization. Eur J Pharmacol 677: 47-54, 2012.

14. Li T, Su L, Zhong N, Hao X, Zhong D, Singhal S and Liu X: Salinomycin induces cell death with autophagy through activation of endoplasmic reticulum stress in human cancer cells. Autophagy 9: 1057-1068, 2013.

15. Ouyang L, Shi Z, Zhao S, Wang FT, Zhou TT, Liu B and Bao JK: Programmed cell death pathways in cancer: A review of apoptosis, autophagy and programmed necrosis. Cell Prolif 45: 487-498, 2012

16. Liu YP, Li L, Xu L, Dai EN and Chen WD: Cantharidin suppresses cell growth and migration, and activates autophagy in human non-small cell lung cancer cells. Oncol Lett 15: 6527-6532, 2018

17. White E: The role for autophagy in cancer. J Clin Invest 125 : 42-46, 2015

18. Parzych KR and Klionsky DJ: An overview of autophagy: morphology, mechanism, and regulation. Antioxid Redox Signal 20: 460-473, 2014

19. Fan S, Qi M, Yu Y, Li L, Yao G, Tashiro S, Onodera S and Ikejima T: P53 activation plays a crucial role in silibinin induced ROS generation via PUMA and JNK. Free Radic Res 46: 310-319, 2012

20. Kuntz S, Wenzel U and Daniel H: Comparative analysis of the effects of flavonoids on proliferation, cytotoxicity, and apoptosis in human colon cancer cell lines. Eur J Nutr 38: 133-142, 1999

21. Bonavida B, Ng C, Jazirehi A, Schiller G and Mizutani Y Selectivity of TRAIL-mediated apoptosis of cancer cells and synergy with drugs: The trail to non-toxic cancer therapeutics. Int J Oncol 15: 793-1595, 1999.

22. Ji D, Zhang Z, Cheng L, Chang J, Wang S, Zheng B, Zheng R, Sun Z, Wang C, Zhang Z, et al: The combination of RAD001 and MK-2206 exerts synergistic cytotoxic effects against PTEN mutant gastric cancer cells: Involvement of MAPK-dependent autophagic, but not apoptotic cell death pathway. PLoS One 9: e85116, 2014.

23. Nazim UM, Moon JH, Lee JH, Lee YJ, Seol JW, Eo SK, Lee JH and Park SY: Activation of autophagy flux by metformin downregulates cellular FLICE-like inhibitory protein and enhances TRAIL-induced apoptosis. Oncotarget 7: 23468-23481, 2016

24. Labsch S, Liu L, Bauer N, Zhang Y, Aleksandrowicz E, Gladkich J, Schönsiegel F and Herr I: Sulforaphane and TRAIL induce a synergistic elimination of advanced prostate cancer stem-like cells. Int J Oncol 44: 1470-1480, 2014.

25. Lagadec C, Adriaenssens E, Toillon R, Chopin V, Romon R, Van Coppenolle F, Hondermarck $\mathrm{H}$ and Le Bourhis X: Tamoxifen and TRAIL synergistically induce apoptosis in breast cancer cells. Oncogene 27: 1472-1477, 2008.

26. Zhu H, Ding WJ, Wu R, Weng QJ, Lou JS, Jin RJ, Lu W, Yang B and $\mathrm{He}$ QJ: Synergistic anti-cancer activity by the combination of TRAIL/APO-2L and celastrol. Cancer Invest 28: 23-32, 2010.

27. Oh YT, Yue P, Wang D, Tong JS, Chen ZG, Khuri FR and Sun SY: Suppression of death receptor 5 enhances cancer cell invasion and metastasis through activation of caspase-8/TRAF2-mediated signaling. Oncotarget 6: 41324-41338, 2015.

28. Han B, Yao W, Oh YT, Tong JS, Li S, Deng J, Yue P, Khuri FR and Sun SY: The novel proteasome inhibitor carfilzomib activates and enhances extrinsic apoptosis involving stabilization of death receptor 5. Oncotarget 6: 17532-17542,2015.
29. von Karstedt S, Montinaro A and Walczak H: Exploring the TRAILs less travelled: TRAIL in cancer biology and therapy. Nature reviews. Cancer 17: 352-366, 2017.

30. Selvarajoo K: A systems biology approach to overcome TRAIL resistance in cancer treatment. Prog Biophys Mol Biol 128: 142-154, 2017.

31. Poornima P, Weng CF and Padma VV: Neferine from Nelumbo nucifera induces autophagy through the inhibition of $\mathrm{PI} 3 \mathrm{~K} / \mathrm{Akt} / \mathrm{mTOR}$ pathway and ROS hyper generation in A549 cells. Food Chem 141: 3598-3605, 2013.

32. Klionsky DJ and Emr SD: Autophagy as a regulated pathway of cellular degradation. Science 290: 1717-1721, 2000.

33. Petersen A, Larsen KE, Behr GG, Romero N, Przedborski S, Brundin P and Sulzer D: Expanded CAG repeats in exon 1 of the Huntington's disease gene stimulate dopamine-mediated striatal neuron autophagy and degeneration. Hum Mol Genet 10: 1243-1254, 2001

34. Kim SW, Lee JH, Moon JH, Nazim UM, Lee YJ, Seol JW, Hur J, Eo SK, Lee JH and Park SY: Niacin alleviates TRAIL-mediated colon cancer cell death via autophagy flux activation. Oncotarget 7: 4356-4368, 2016.

35. Zhang Y, Wu Y, Cheng Y, Zhao Z, Tashiro S, Onodera S and Ikejima T: Fas-mediated autophagy requires JNK activation in HeLa cells. Biochem Biophys Res Commun 377: 1205-1210, 2008.

36. Stuckey DW and Shah K: TRAIL on trial: Preclinical advances in cancer therapy. Trends Mol Med 19: 685-694, 2013

37. Xu L, Zhang X, Li Y, Lu S, Lu S, Li J, Wang Y, Tian X, Wei JJ, Shao C and Liu Z: Neferine induces autophagy of human ovarian cancer cells via p38 MAPK/JNK activation. Tumour Biol 37: 8721-8729, 2016.

38. Musumeci G, Castrogiovanni P, Trovato FM, Weinberg AM, Al-Wasiyah MK, Alqahtani MH and Mobasheri A: Biomarkers of chondrocyte apoptosis and autophagy in osteoarthritis. Int J Mol Sci 16: 20560-20575, 2015.

39. Fiandalo MV and Kyprianou N: Caspase control: Protagonists of cancer cell apoptosis. Exp Oncol 34: 165-175, 2012.

40. Huang K: Mechanism of Bax/Bak Activation in Apoptotic Signaling (unpublished $\mathrm{PhD}$ thesis). University of Nebraska Medical Center, 2019.

41. Ly JD, Grubb DR and Lawen A: The mitochondrial membrane potential (deltapsi(m)) in apoptosis; an update. Apoptosis 8: $115-128,2003$.

42. Tounekti O, Belehradek J Jr and Mir L: Relationships between DNA fragmentation, chromatin condensation, and changes in flow cytometry profiles detected during apoptosis. Exp Cell Res 217: 506-516, 1995

43. Yoshii SR and Mizushima N: Monitoring and measuring autophagy. Int J Mol Sci 18: 1865, 2017.

44. Tanida I, Ueno T and Kominami E: LC3 and autophagy. Methods Mol Biol 445: 77-88, 2008

45. Ganesher A, Chaturvedi P, Sahai R, Meena S, Mitra K, Datta D and Panda G: New spisulosine derivative promotes robust autophagic response to cancer cells. Eur J Med Chem 188: 112011,2020

46. Han X, Guo L, Jiang X, Wang Y, Wang Z and Li D: Curcumin inhibits cell viability by inducing apoptosis and autophagy in human colon cancer cells. Proceed Anticancer Res 3: 21-25, 2019.

47. Chiou JT, Huang CH, Lee YC, Wang LJ, Shi YJ, Chen YJ and Chang LS: Compound $\mathrm{C}$ induces autophagy and apoptosis in parental and hydroquinone-selected malignant leukemia cells through the ROS/p38 MAPK/AMPK/TET2/FOXP3 axis. Cell Biol Toxicol: Jan 3, 2020 (Epub ahead of prin).

48. Opipari AW Jr, Tan L, Boitano AE, Sorenson DR, Aurora A and Liu JR: Resveratrol-induced autophagocytosis in ovarian cancer cells. Cancer Res 64: 696-703, 2004.

This work is licensed under a Creative Commons Attribution-NonCommercial-NoDerivatives 4.0 International (CC BY-NC-ND 4.0) License. 